\title{
El rol de las redes sociales en la difusión de la identidad corporativa de los hospitales españoles
}

The role of social media networks in disseminating the corporate identity of Spanish hospitals

Antonio González Pacanowski, Universidad de Alicante - toni.gonzalez@ua.es

Pablo Medina Aguerrebere, Universidad de Ginebra (Suiza) - pablo.medina@unige.ch

\section{Resumen}

La consolidación de las redes sociales como herramienta de comunicación institucional, las nuevas expectativas de los pacientes, y el mayor interés de la gente hacia la salud ha obligado a los hospitales a repensar sus estrategias de comunicación online, especialmente las relativas a la difusión de la identidad corporativa. El objetivo de este artículo es analizar el rol de las redes sociales en la difusión de la identidad corporativa de los hospitales. Para ello, se realiza una revisión bibliográfica sobre los conceptos de comunicación institucional, marca, identidad y redes sociales; y, posteriormente, se analiza el uso de las redes sociales por parte de los hospitales españoles. La conclusión del artículo es que los hospitales deben fomentar la formación del profesional de la salud en comunicación institucional, la reflexión estratégica y el uso integrado de redes sociales y web propia.

\section{Palabras clave}

Hospital, comunicación institucional, marca, identidad, redes sociales.

\section{Abstract}

The implementation of social media as a tool of corporate communication, new expectations of patients and the greater interest of people towards health has lead hospitals to rethink their online communication strategies, especially those related to the dissemination of corporate identity. This paper aims to analyze the role of social media in the dissemination of corporate identity of hospitals. To do this, we made a literature review about corporate communication, brand, identity and social media; and, subsequently, we analyzed the use of social media by Spanish hospitals. This paper concludes that hospitals should promote the training of health professionals in corporate communication, the strategic reflection and the integrated use of social media and their own website.

\section{Keywords}

Hospital, corporate communication, brand, identity, social media.

\section{Sumario}

1. Introducción. 2. La marca hospitalaria. 3. La marca hospitalaria en el entorno 2.0. 4. Metodología. 5. Resultados y discusión. 6. Conclusión. 7. Referencias bibliográficas. 


\section{Introducción}

La consolidación de las redes sociales y de las aplicaciones móviles como herramientas de comunicación institucional ha llevado a varios hospitales a repensar sus estrategias de comunicación online con el objetivo de mejorar su visibilidad de marca y reforzar así su posicionamiento estratégico en el mercado. Este entorno digital plantea varios retos a los hospitales, como por ejemplo la existencia de un gran número de redes sociales, la posibilidad de usar diferentes formatos (texto, imagen, vídeo, infografía, etc.), la necesidad de implicar al profesional de la salud en las acciones de comunicación online e incluso el monitoreo de las conversaciones mantenidas en las redes sociales que el hospital gestiona. En este contexto, surge una pregunta: ¿qué impacto tienen las redes sociales en la difusión de la identidad corporativa del hospital? El objetivo de este artículo es analizar la comunicación institucional que llevan a cabo los hospitales españoles para difundir su identidad corporativa a través de las redes sociales. Para ello, en primer lugar, se realiza una revisión bibliográfica sobre los conceptos de comunicación institucional, marca, identidad y redes sociales; $y$, en segundo lugar, se analizan las estrategias de comunicación de marca que utilizan los principales hospitales de España en las redes sociales.

\section{La marca hospitalaria}

En el entorno hospitalario, se pueden identificar tres actividades comunicativas diferentes: comunicación interpersonal, comunicación interna y comunicación externa. La primera hace referencia a la comunicación establecida entre el profesional de la salud y el paciente; dicha comunicación repercute en los resultados físicos y psicológicos que este último obtiene con el tratamiento médico, así como en los posibles errores que el profesional de la salud pueda cometer a la hora de realizar el diagnóstico (Wright, Sparks y O'Hair, 2008). La comunicación interna, por su parte, se refiere a todas las interacciones comunicativas que mantienen entre sí los empleados de un hospital para preparar el servicio médico solicitado por el paciente (Berry, 2007). En cuanto a la comunicación externa, se trata de las distintas actividades de comunicación que lleva a cabo el hospital para establecer relaciones satisfactorias con sus diferentes stakeholders - pacientes, autoridades sanitarias, medios de comunicación, etc.- (Medina, 2011). La gestión coherente y estratégica de estas tres actividades comunicativas dan lugar a la comunicación institucional hospitalaria. Según Van Riel y Fombrun (2007: 25), la comunicación institucional es "el conjunto de actividades incluidas en la gestión y en la organización de todas las comunicaciones internas y externas cuyo objetivo es crear puntos de partida favorables respecto a los grupos de interés de los que depende la compañía".

El Director de Comunicación del hospital asume la responsabilidad de gestionar de un modo estratégico y planificado todas las actividades de comunicación institucional con el objetivo de construir una marca sólida y reputada que ayude a la organización hospitalaria a mantener buenas relaciones con sus stakeholders y así consolidar su posicionamiento estratégico en el mercado. En el contexto hospitalario actual, caracterizado por las nuevas exigencias y expectativas de los pacientes, así como por el rol comunicativo cada vez más importante que asumen los empleados del hospital, resulta especialmente necesario que la marca corporativa se convierta en el activo intangible más importante del que dispone la organización (Naveen, Anil y Smruthi, 2014). La marca no es un concepto abstracto, sino un grupo de valores emocionales y funcionales que permiten hacer realidad la promesa de la organización (De Chernatony, 2010). El desarrollo de una estrategia de marca que ayude al hospital a establecer y mantener relaciones satisfactorias con sus principales stakeholders constituye una prioridad estratégica ya que la marca ayuda a dichas organizaciones a adaptarse al cambio constante al que están sometidas (Kemp, Jilipalli y Becerra, 2014).

A la hora de construir su marca, los hospitales pueden elegir entre tres modelos diferentes: a) modelo monolítico, en el que se da todo el peso a la marca de la organización; b) modelo refrendo, que consiste en dar más importancia a las marcas secundarias (departamentos médicos, centros adheridos al hospital, etc.) que a la marca global; o c) modelo mixto, que se basa en otorgar el mismo peso a todas las marcas (Buckley, 2007). Una vez elegido el modelo de marca, el Director de Comunicación del hospital, en colaboración con los directivos del mismo, debe definir la arquitectura de marca de la organización: identidad, valores, misión, visión y cultura. La identidad hace referencia a la esencia de una organización y constituye un eje estratégico que determina el funcionamiento de la misma (Abratt y Kleyn, 2012). Los valores son elementos concretos en los que se plasma la identidad y que están siempre presentes en los comportamientos y en los procesos de trabajo que tienen lugar en la organización (Jaakson, 2010). La misión hace referencia a los objetivos perseguidos por una organización y se plasma en ideas concretas que ayudan a los empleados a llevar a la práctica dicha misión (Cady et al., 2011). La visión tiene un componente de más largo plazo y se refiere a los objetivos que persigue la organización en el largo plazo, así como a los cambios necesarios para alcanzar dichos objetivos (Singal y Jain, 2013). Por último, la cultura queda determinada por la capacidad del hospital para alinear la misión, los valores y las prácticas profesionales de cada día para así lograr que la forma de funcionar de dicho hospital sea única e incomparable con la de ningún otro competidor (Nelson, Taylor y Walsh, 2014).

\section{La marca hospitalaria en el entorno 2.0}

En el entorno hospitalario, las redes sociales se han consolidado como una verdadera herramienta de comunicación institucional. Gracias a ellas, los pacientes pueden buscar y compartir información médica, recibir apoyo emocional, formarse en temas médicos e incluso comunicarse con los profesionales de la salud para solucionar dudas relativas a tratamientos o patologías. Las redes sociales han puesto en evidencia el enorme conocimiento sobre temas médicos que reside en las distintas colectividades de pacientes (Metzger y Flanagin, 2011). Por su parte, el profesional de la salud usa dichas redes para difundir información médica al paciente, pero también para aumentar el compromiso de dicho paciente con el diseño del tratamiento médico, el proceso de toma de decisiones colectivas -tratamiento, uso de medicamentos, etc.- y la evaluación de la mejora continua (Fordis et al., 2011). Las redes sociales se han convertido en una herramienta estratégica que mejora las relaciones comunicativas, médicas, culturales y sociales que establecen los profesionales de la salud que trabajan en un hospital con los pacientes y sus familiares.

Sin embargo, las redes sociales no suponen beneficios únicamente para el paciente y para el profesional de la salud; el hospital, como institución, también puede aprovechar las numerosas ventajas que ofrecen las redes sociales como herramienta de comunicación institucional. Entre dichas ventajas, destacan cinco. En primer lugar, las redes sociales facilitan la gestión de ciertos problemas de salud pública, lo cual ayuda a legitimar aún más el rol de la comunicación institucional como un área estratégica en las organizaciones sanitarias (Salathé et al., 2013). En segundo lugar, gracias a las redes sociales, los hospitales pueden mejorar la comunicación interna con los empleados, lo cual facilita la construcción colectiva de la marca y, por tanto, la atracción de pacientes, empleados y proyectos de investigación (Thielst, 2011). En tercer lugar, el recurso a las redes sociales ayuda a los profesionales de la salud que trabajan en un centro hospitalario a mejorar sus relaciones comunicativas con el paciente, lo cual repercute 
positivamente en la calidad percibida por este último (The Change Foundation, 2011). En cuarto lugar, la información difundida por los pacientes y los profesionales de la salud en las redes sociales gestionadas por el hospital queda indexada en distintos motores de búsqueda, lo cual permite mejorar la visibilidad de la marca hospitalaria en Internet (Van de Belt et al., 2012). Y en quinto lugar, el número de redes sociales en las que está presente el hospital, la información médica e institucional que en ellas difunde y el tipo de interacción mantenida con los pacientes influyen positivamente en la imagen de marca del hospital (Huesch, Currid-Halkett y Doctor, 2014).

Las redes sociales constituyen una herramienta imprescindible para todas las organizaciones hospitalarias que desean potenciar su marca. En el entorno digital, la promoción de la marca -ya sea la marca corporativa del hospital, la de sus departamentos médicos o centros adheridos, o la de sus profesionales de la salud-constituye una prioridad estratégica ya que ayuda a los hospitales a reforzar su posicionamiento en el mercado. En el contexto hospitalario, se pueden identificar diez razones que justifican el rol estratégico de las redes sociales en la promoción de la marca.

En primer lugar, las redes sociales facilitan la difusión de información médica al paciente, lo que permite legitimar la autoridad científica del hospital (Fernández-Luque y Bau, 2015). En segundo lugar, la difusión en las redes sociales de videos sobre contenido médico ayuda al paciente a interiorizar conceptos médicos especialmente complicados, lo que repercute en su satisfacción con la organización hospitalaria (Gabarrón et al., 2013). En tercer lugar, las redes sociales ayudan a los hospitales a difundir información institucional adaptada a las necesidades de los distintos stakeholders, lo cual permite que éstos mejoren sus percepciones sobre la organización (Centers for Disease Control and Prevention, 2012). En cuarto lugar, gracias a las redes sociales, los hospitales pueden fomentar el boca a oreja positivo entre médicos y pacientes para así favorecer la construcción colectiva de la marca (McCarroll et al., 2014). En quinto lugar, las redes sociales constituyen una herramienta muy apropiada para fomentar la implicación del paciente con el profesional de la salud y mejorar así la relación médica y comunicativa que se establece entre ambos (Visser et al., 2016).

En sexto lugar, la información difundida por los pacientes en las redes sociales permite al hospital comprender mejor sus percepciones sobre la organización y adaptar así la comunicación de marca para que ésta sea más eficaz (Alemi et al., 2012). En séptimo lugar, la comunicación del hospital a través de las redes sociales permite potenciar el resto de actividades comunicativas que éste lleva a cabo y aumentar así el impacto de marca (Chiu-chi y Khurana, 2012). En octavo lugar, gracias a las redes sociales, los hospitales pueden atraer más pacientes hacia su página web y mejorar así la visibilidad de marca (Huang y Dunbar, 2013). En noveno lugar, el recurso a las redes sociales facilita la difusión y aplicación de la misión corporativa del hospital ante sus diferentes grupos de interés (Thaker et al., 2011). Y, en décimo lugar, las redes sociales constituyen una plataforma oportuna para mejorar la evaluación y gestión de la reputación de marca (Würz, Nurm y Ekdahl, 2013).

En el entorno hospitalario, las redes sociales se han convertido en una herramienta muy potente para difundir la identidad corporativa y así mejorar las relaciones del hospital con sus stakeholders. En las redes sociales, la identidad de marca se crea y difunde a través de la información disponible en los perfiles corporativos de las organizaciones; por eso, resulta tan importante que éstas cuiden la información biográfica y los elementos visuales disponibles en dichos perfiles, ya que los mismos reflejan las características de las organizaciones y determinan las percepciones que sobre éstas tiene los stakeholders (Scott, 2010). Independientemente de la red social de la que se trate, la información corporativa difundida por los hospitales debe ser lo suficientemente precisa como para que todos los stakeholders comprendan la esencia de la marca hospitalaria. La difusión de la identidad de marca a través de las redes sociales ayuda a la organización a mejorar su visibilidad y reconocimiento (Fanion, 2011) así como a establecer relaciones satisfactorias con sus diferentes stakeholders, lo cual es clave para alcanzar los objetivos globales de comunicación institucional perseguidos por la organización (Park, Rodgers y Stemmle, 2011).

La gestión de la identidad digital constituye una prioridad estratégica para todos los hospitales. Sin embargo, antes de difundir ninguna información en las redes sociales, los hospitales deben realizar una reflexión estratégica que les permita definir correctamente los elementos integrantes de su arquitectura de marca (identidad, valores, misión, visión y cultura) así como establecer una estrategia de comunicación online para difundir eficazmente dichos elementos a los diferentes stakeholders. Además, y como paso previo a la ejecución de dicha estrategia, los hospitales deben solucionar algunos de los problemas que plantea el uso de las redes sociales como herramienta de comunicación institucional, como por ejemplo la privacidad, la seguridad, la gestión de la identidad y la usabilidad (Househ, Borycki y Kushniruk, 2014). Una vez realizada dicha reflexión estratégica, los hospitales deben formar a sus profesionales de la salud en habilidades de comunicación online para que logren transmitir correctamente la identidad del hospital a los distintos stakeholders con los que interactúan, especialmente con los pacientes. Para responder eficazmente a las nuevas necesidades comunicativas de los pacientes, los hospitales necesitan profesionalizar su comunicación en redes sociales (Lee et al., 2015), y para ello resulta fundamental contar con la colaboración del principal comunicador de dichas organizaciones: el profesional de la salud. La realización de una reflexión estratégica y la formación del profesional de la salud constituyen los dos pilares sobre los que se basa la correcta difusión de la identidad de marca hospitalaria en el entorno digital.

\section{Metodología}

Atendiendo a la información disponible en el Catálogo Nacional de Hospitales publicado por el Ministerio de Sanidad, Política Social e Igualdad en 2017, se exponen los datos clasificados bajo las categorías de hospitales privados con red social en España, web propia y con blog. La diferenciación entre público y privado se ha establecido según la responsabilidad pública o privada del centro. La extracción se ha realizado desde la base de datos del grupo de Tecnologías para la salud y Bienestar del Instituto ITACA (ITACA-TSB) de la Universidad Politécnica de Valencia, dado su alto grado de actualización. Igualmente se ha atendido a la clasificación realizada en el Ranking Mundial de Hospitales 2015, elaborado por el Consejo Superior de Investigaciones Científicas de España-CSIC- para confirmar el posicionamiento del tráfico en de las respectivas webs en internet. 


\section{Resultados y discusión}

En la categoría de hospitales privados, el número de centros está vinculado a la frecuencia de redes sociales en la Comunidad Autónoma. Así, las comunidades de Cataluña (32), Andalucía (29) y Madrid (17), con mayor oferta de centros privados, encabezan el ranking bajo este concepto (ver Tabla 1. Hospitales privados con red social en España). No obstante, Andalucía destaca por su proporción más elevada frente a la segunda. Un $49,15 \%$ frente a un $19.88 \%$. Respecto a los hospitales públicos, el mayor número de redes sociales se constata por orden en la Comunidad Valenciana (24), Cataluña (20) y Andalucía -15- (ver Tabla 2. Hospitales públicos con red social en España). Comparativamente, la red privada (148) dispone de una estructura de redes sociales más numerosa que la pública (84).

Por tipo de red social en centros privados, una revisión de las redes más empleadas constata que Facebook la más frecuente (103), seguida de Twitter (102), Youtube (80), Wikipedia (75), Google+ (41), Linkedin (27) e Instagram (4)-ver. Imagen 1. Redes sociales más frecuentes en hospitales privados-. Sin embargo, en los centros públicos, Twitter es la más empleada (68), seguida de Facebook (40), Youtube (33), Wikipedia (16), Google+ (8), Linkedin (5) e Instagram (4) -ver Imagen 2. Redes sociales más frecuentes en hospitales públicos-.

Imagen 1. Redes sociales más frecuentes en hospitales privados

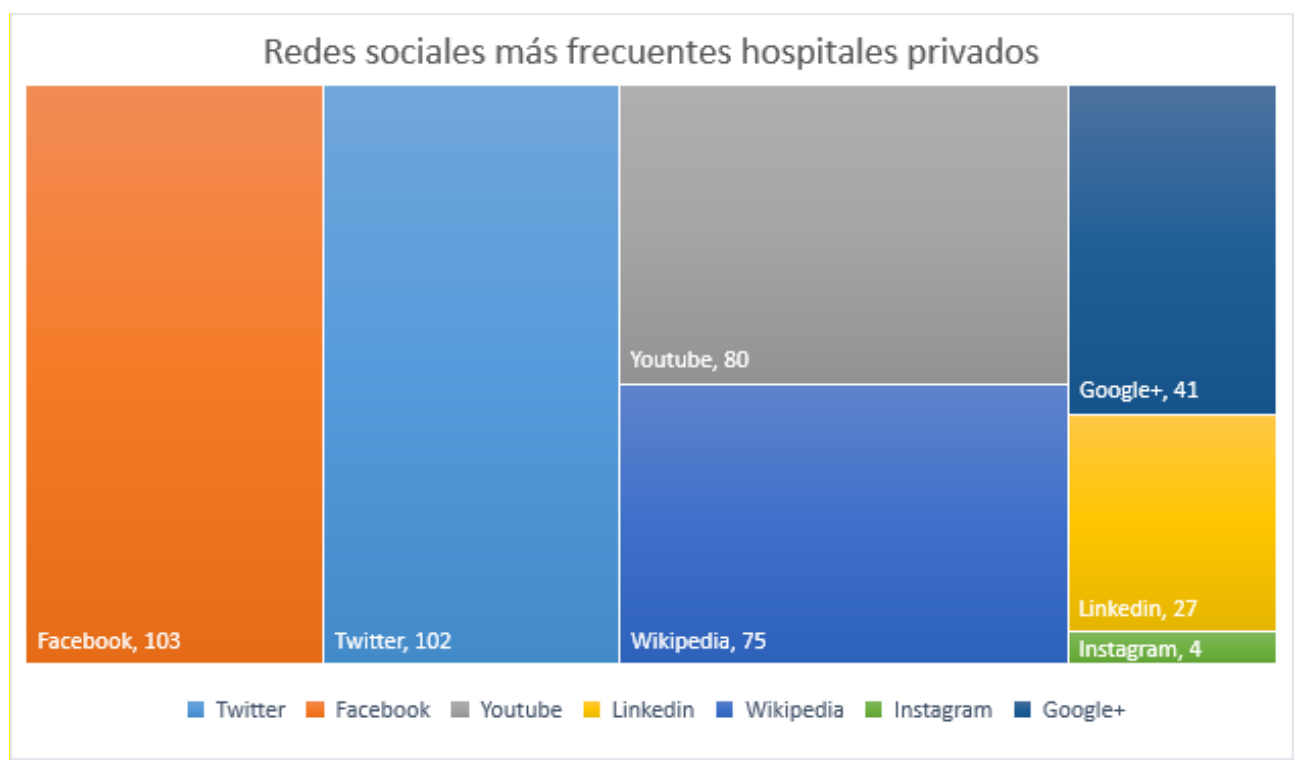

Fuente: Elaboración propia

Imagen 2. Redes sociales más frecuentes en hospitales públicos

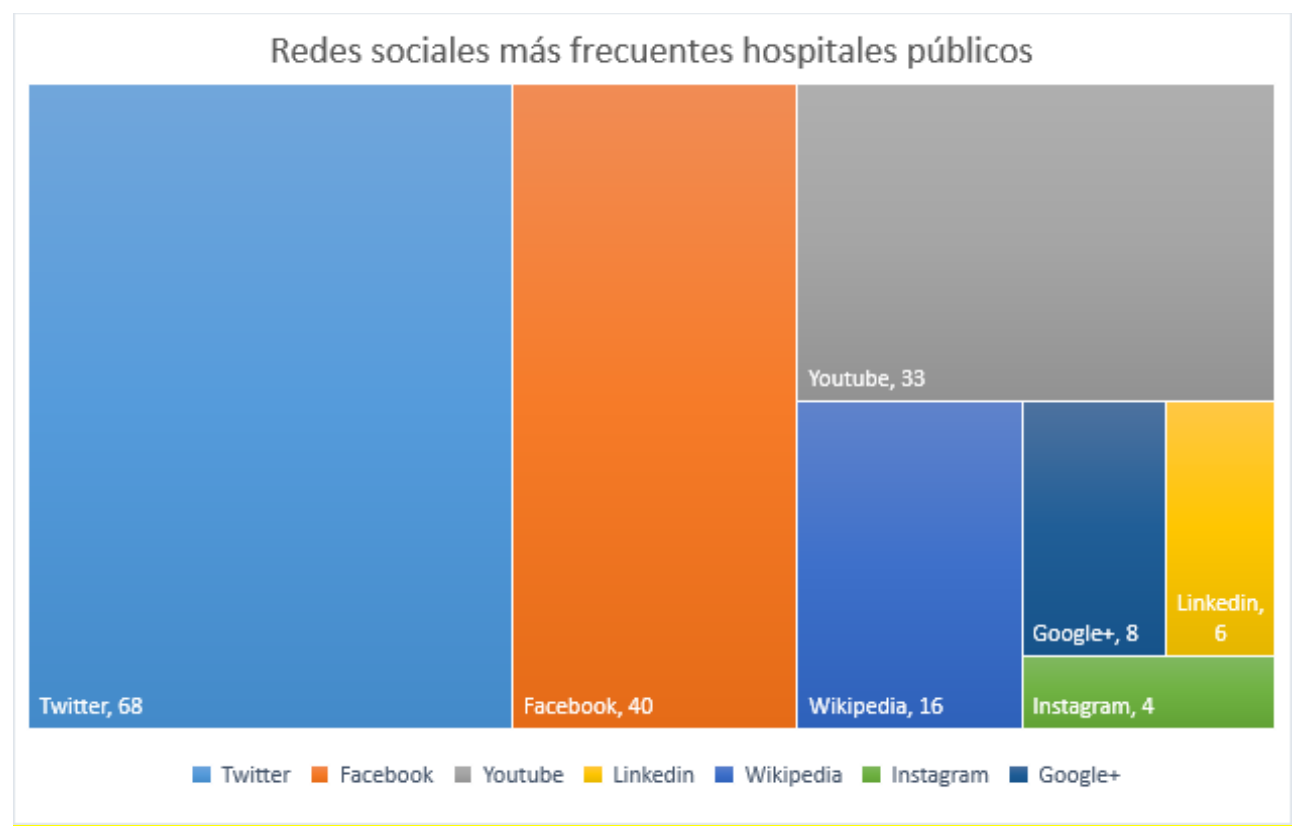

Fuente: Elaboración propia 
Tabla 1. Hospitales privados con red social en España

\begin{tabular}{lccc}
\hline Comunidad Autónoma & $\mathbf{T}$ & $\%$ & $\mathbf{N}^{\mathbf{0}}$ \\
\hline Andalucía & 59 & 49.15 & 29 \\
\hline Aragón & 10 & 30 & 3 \\
\hline Asturias & 11 & 18.18 & 2 \\
\hline Canarias & 24 & 29.17 & 7 \\
\hline Cantabria & 4 & 25 & 1 \\
\hline Castilla y León & 21 & 28.57 & 6 \\
\hline Castilla-La Mancha & 9 & 0 & 0 \\
\hline Cataluña & 161 & 19.88 & 32 \\
\hline Ceuta & 1 & 100 & 1 \\
\hline Comunidad Valenciana & 26 & 46.15 & 12 \\
\hline Extremadura & 11 & 9.09 & 1 \\
\hline Galicia & 24 & 54.17 & 13 \\
\hline Islas Baleares & 13 & 53.85 & 7 \\
\hline La Rioja & 3 & 33.33 & 1 \\
\hline Madrid & 47 & 36.17 & 17 \\
\hline Melilla & 1 & 0 & 0 \\
\hline Murcia & 16 & 43.75 & 7 \\
\hline Navarra & 7 & 28.57 & 2 \\
\hline País Vasco & 24 & 29.17 & 7 \\
\hline Total & 472 & 31.36 & 148 \\
\hline & & \\
\hline T: Total centros por Comunidad Autónoma & & \\
\hline \%: Porcentaje centros con redes sociales por & & \\
Comunidad Autónoma & & \\
\hline No: Número de centros con redes sociales por Comunidad Autónoma & \\
\hline
\end{tabular}

Fuente: Elaboración propia

Tabla 2. Hospitales públicos con red social en España

\begin{tabular}{lccc}
\hline Comunidad Autónoma & $\mathbf{T}$ & $\%$ & $\mathbf{N}^{\mathbf{0}}$ \\
\hline Andalucía & 43 & 34.88 & 15 \\
\hline Aragón & 19 & 0 & 0 \\
\hline Asturias & 9 & 11.11 & 1 \\
\hline Canarias & 15 & 0 & 0 \\
\hline Cantabria & 3 & 33.33 & 1 \\
\hline Castilla y Léon & 15 & 0 & 0 \\
\hline Castilla-La Mancha & 20 & 25 & 5 \\
\hline Cataluña & 50 & 40 & 20 \\
\hline Ceuta & 1 & 0 & 0 \\
\hline Comunidad Valenciana & 35 & 68.57 & 24 \\
\hline Extremadura & 8 & 0 & 0 \\
\hline Galicia & 16 & 6.25 & 1 \\
\hline Islas Baleares & 11 & 54.55 & 6 \\
\hline La Rioja & 3 & 0 & 0 \\
\hline Madrid & 33 & 21.21 & 7 \\
\hline Melilla & 0 & 0 & 0 \\
\hline Murcia & 10 & 10 & 1 \\
\hline Navarra & 6 & 0 & 0 \\
\hline País Vasco & 18 & 16.67 & 3 \\
\hline Total & 315 & 26.67 & 84 \\
\hline \multicolumn{4}{l}{ Total centros por Comunidad. Autónoma } \\
\hline \%: Porcentaje centros con redes sociales por Comunidad Autónoma \\
\hline No Número de centros con redes sociales por Comunidad Autónoma \\
\hline
\end{tabular}

Fuente: Elaboración propia 
Respecto a los centros públicos con web propia, Cataluña es la primera (29), obviamente debido al número de centros superior como elemento potenciador. No obstante, comunidades como el País Vasco, Madrid, o Castilla-León disponen de web propia en la mayoría de centros, lo que contrasta con el caso catalán, donde el porcentaje de web de hospitales que disponen de web propia es del 58\% (ver tabla 3. Hospitales públicos con web propia). En la red privada, Cataluña lidera el número de centros con web propia (74), seguida de Andalucía (41) y Madrid (36) - ver Tabla 4. Hospitales privados con web propia-. En número absolutos, existe mayor número de centros privados (282) con web propia que públicos (182), lo que también se explica por la oferta superior de centros hospitalarios privados (472) frente a los públicos (315). En cuanto al uso de Blog, entendido como una web independiente de la corporativa, existe mayor presencia de dicho recurso en la red privada (30) que en la pública (18), lo que también obedece, como sucede en la web propia, a la cantidad superior de oferta privada de centros (ver Tabla 5. Centros privados con blog en España y Tabla 6. Centros públicos con blog en España).

Tabla 3. Hospitales públicos con web propia

\begin{tabular}{lccc}
\hline Comunidad Autónoma & $\mathbf{T}$ & $\%$ & $\mathbf{N}^{\mathbf{0}}$ \\
\hline Andalucía & 43 & 53.49 & 23 \\
\hline Aragón & 19 & 26.32 & 5 \\
\hline Asturias & 9 & 66.67 & 6 \\
\hline Canarias & 15 & 13.33 & 2 \\
\hline Cantabria & 3 & 100 & 3 \\
\hline Castilla y Léon & 15 & 93.33 & 14 \\
\hline Castilla-La Mancha & 20 & 75 & 15 \\
\hline Cataluña & 50 & 58 & 29 \\
\hline Ceuta & 1 & 0 & 0 \\
\hline Comunidad Valenciana & 35 & 65.71 & 23 \\
\hline Extremadura & 8 & 0 & 0 \\
\hline Galicia & 16 & 25 & 4 \\
\hline Islas Baleares & 11 & 45.45 & 5 \\
\hline La Rioja & 3 & 33.33 & 1 \\
\hline Madrid & 33 & 93.94 & 31 \\
\hline Melilla & 0 & 0 & 0 \\
\hline Murcia & 10 & 30 & 3 \\
\hline Navarra & 6 & 16.67 & 1 \\
\hline País Vasco & 18 & 94.44 & 17 \\
\hline Total & 315 & 57.78 & 182 \\
\hline & \multicolumn{3}{l}{} \\
\hline T: Total centros por Comunidad Autónoma & \\
\hline \%: Porcentaje centros con webs propias por Comunidad Autónoma & \\
\hline No: Número de centros con webs propias por Comunidad Autónoma & \\
\hline
\end{tabular}

Fuente: Elaboración propia

Tabla 4. Hospitales privados con web propia

\begin{tabular}{lccc}
\hline Comunidad Autónoma & $\mathbf{T}$ & $\%$ & $\mathbf{N}^{\mathbf{0}}$ \\
\hline Andalucía & 59 & 69.49 & 41 \\
\hline Aragón & 10 & 50 & 5 \\
\hline Asturias & 11 & 72.73 & 8 \\
\hline Canarias & 24 & 41.67 & 10 \\
\hline Cantabria & 4 & 75 & 3 \\
\hline Castilla y Léon & 21 & 76.19 & 16 \\
\hline Castilla-La Mancha & 9 & 55.56 & 5 \\
\hline Cataluña & 161 & 45.96 & 74 \\
\hline Ceuta & 1 & 100 & 1 \\
\hline Comunidad Valenciana & 26 & 65.38 & 17 \\
\hline Extremadura & 11 & 36.36 & 4 \\
\hline Galicia & 24 & 75 & 18 \\
\hline Islas Baleares & 13 & 61.54 & 8 \\
\hline La Rioja & 3 & 66.67 & 2 \\
\hline Madrid & 47 & 76.6 & 36 \\
\hline Melilla & 1 & 100 & 1 \\
\hline Murcia & 16 & 81.25 & 13 \\
\hline
\end{tabular}




\begin{tabular}{cccc}
\hline Navarra & 7 & 57.14 & 4 \\
\hline País Vasco & 24 & 66.67 & 16 \\
\hline Total & 472 & 59.75 & 282 \\
\hline T: Total centros por Comunidad Autónoma \\
\hline \%: Porcentaje centros con webs propias por Comunidad Autónoma \\
\hline No: Número de centros con webs propias por Comunidad Autónoma \\
\hline
\end{tabular}

Fuente: Elaboración propia

Tabla 5. Centros privados con blog en España

\begin{tabular}{lccc}
\hline Comunidad Autónoma & $\mathbf{T}$ & $\%$ & $\mathbf{N}^{\mathbf{0}}$ \\
\hline Andalucía & 59 & 10.17 & 6 \\
\hline Aragón & 10 & 0 & 0 \\
\hline Asturias & 11 & 9.09 & 1 \\
\hline Canarias & 24 & 8.33 & 2 \\
\hline Cantabria & 4 & 25 & 1 \\
\hline Castilla y Léon & 21 & 4.76 & 1 \\
\hline Castilla-La Mancha & 9 & 0 & 0 \\
\hline Cataluña & 161 & 6.83 & 11 \\
\hline Ceuta & 1 & 0 & 0 \\
\hline Comunidad Valenciana & 26 & 0 & 0 \\
\hline Extremadura & 11 & 0 & 0 \\
\hline Galicia & 24 & 8.33 & 2 \\
\hline Islas Baleares & 13 & 0 & 0 \\
\hline La Rioja & 3 & 0 & 0 \\
\hline Madrid & 47 & 6.38 & 3 \\
\hline Melilla & 1 & 0 & 0 \\
\hline Murcia & 16 & 18.75 & 3 \\
\hline Navarra & 7 & 0 & 0 \\
\hline País Vasco & 24 & 0 & 0 \\
\hline Total & 472 & 6.36 & 30 \\
\hline & \multicolumn{4}{l}{} \\
\hline T: Total centros por Comunidad Autónoma \\
\hline \%: Porcentaje centros con webs propias por Comunidad Autónoma \\
\hline No: Número de centros con webs propias por Comunidad Autónoma \\
\hline
\end{tabular}

Fuente: Elaboración propia

Tabla 6. Centros públicos con blog en España

\begin{tabular}{lccc}
\hline Comunidad Autónoma & $\mathbf{T}$ & $\%$ & $\mathbf{N}^{\mathbf{0}}$ \\
\hline Andalucía & 43 & 11.63 & 5 \\
\hline Aragón & 19 & 10.53 & 2 \\
\hline Asturias & 9 & 11.11 & 1 \\
\hline Canarias & 15 & 0 & 0 \\
\hline Cantabria & 3 & 0 & 0 \\
\hline Castilla y Léon & 15 & 0 & 0 \\
\hline Castilla-La Mancha & 20 & 0 & 0 \\
\hline Cataluña & 50 & 10 & 5 \\
\hline Ceuta & 1 & 0 & 0 \\
\hline Comunidad Valenciana & 35 & 11.43 & 4 \\
\hline Extremadura & 8 & 0 & 0 \\
\hline Galicia & 16 & 6.25 & 1 \\
\hline Islas Baleares & 11 & 0 & 0 \\
\hline La Rioja & 3 & 0 & 0 \\
\hline Madrid & 33 & 0 & 0 \\
\hline
\end{tabular}




\begin{tabular}{lccc}
\hline Melilla & 0 & 0 & 0 \\
\hline Murcia & 10 & 0 & 0 \\
\hline Navarra & 6 & 0 & 0 \\
\hline País Vasco & 18 & 0 & 0 \\
\hline Total & 315 & 5.71 & 18 \\
\hline \multicolumn{4}{l}{} \\
\hline T: Total centros por Comunidad Autónoma \\
\hline \%: Porcentaje centros con webs propias por Comunidad Autónoma \\
\hline No: Número de centros con webs propias por Comunidad Autónoma \\
\hline
\end{tabular}

Fuente: Elaboración propia

\section{Conclusión}

La difusión de la identidad corporativa a través de las redes sociales constituye una oportunidad estratégica para todos aquellos hospitales que desean potenciar su reputación corporativa. Con el objetivo de concluir este artículo, se ofrecen tres últimas ideas. En primer lugar, los hospitales deben apostar por la formación de los profesionales de la salud en técnicas de comunicación institucional para así garantizar que las percepciones que los stakeholders tienen sobre la identidad del hospital sean únicas y coherentes. En segundo lugar, los hospitales deben realizar una reflexión estratégica previa que les permita decidir en cuantas redes sociales van a estar presentes, así como en cuáles de ellas. En el caso analizado en este artículo, resulta evidente que el sector público mantiene proporcionalmente un nivel competitivo de su identidad digital con porcentajes elevados de cumplimiento, y que determinadas redes sociales son mucho más frecuentes en los centros privados, especialmente las de contenido richmedia (Youtube) o impacto en las relaciones profesionales y comerciales (Linkedin), dejando al margen el factor de la oferta superior de centros. Y, en tercer lugar, el comportamiento digital de los hospitales españoles deja entrever que, en los próximos años, las acciones de difusión de la identidad corporativa priorizarán el uso mixto de web propia y red social, para así garantizar la interacción entre el hospital y sus stakeholders, así como la difusión de contenidos corporativos y científicos.

\section{Bibliografía}

Abratt, R. y Kleyn, N. (2012). Corporate identity, corporate branding and corporate reputations. European Journal of Marketing, 46(7/8), 1048-1063. DOI: 10.1108/03p090561211230197

Alemi F., Torii, M., Clement, L. y Aron, D. (2012) Feasibility of real-time satisfaction surveys through automated analysis of patients' unstructured comments and sentiments. Quality Management Health Care, 21, 9-19. DOI: 10.1097/QMH.0b013e3182417fc4

Berry, D. (2007). Health communication: theory and practice. Maidenhead: Open University Press. ISBN: 9780787982058

Buckley, P. (2007). The complete guide to hospital marketing. Marblehead: HCPro Inc. ISBN: 1601463510

Cady, S., Wheeler, J., DeWolf, J. y Brodke, M. (2011). Mission, vision and values: what do they say? Organizational Development Journal, 29(1), 6378.

Centers for Disease Control and Prevention (2012). The health communicator's social media toolkit. Recuperado de http://www.cdc.gov/healthcommunication/ToolsTemplates/SocialMediaToolkit_BM.pdf

Chiu-chi, E. y Khurana, A. (2012). Users' preferred interactive e-health tools on hospital web sites. International Journal of Pharmaceutical and Healthcare Marketing, 6(3),215-229. DOI: http://dx.doi.org/10.1108/17506121211259395

De Chernatony, L. (2010). From brand vision to brand evaluation: the strategic process of growing and strengthening brands. Burlington: Routledge. ISBN: 0750667494

Fanion, R. (2011). Social media brings benefits to top companies. Central Penn Business Journal, 27(3), 76. DOI: 10.1108/MIP-04-2013-0056.

Fernández-Luque, L. y Bau, T. (2015). Health and social media: perfect storm of information. Healthcare Informatics Research, 21(2), 67-73. DOI: http://dx.doi.org/10.4258/hir.2015.21.2.67

Fordis, M., Street, R., Volk, R. y Smith, Q. (2011). The Prospects for Web 2.0 Technologies for Engagement, Communication, and Dissemination in the Era of Patient-Centered Outcomes Research: Selected Articles Developed From the Eisenberg Conference Series 2010 Meeting. Journal of Health Communication, 16(1), 3-9. DOI: 10.1080/10810730.2011.598398

Gabarron, E., Fernandez-Luque, L., Armayones, M. y Lau, A. (2013). Identifying Measures Used for Assessing Quality of YouTube Videos with Patient Health Information: A Review of Current Literature. Interactive Journal of Medical Research, 2(1). DOI: 10.2196/ijmr.2465

Househ, M., Borycki, E. y Kushniruk, A. (2014). Empowering patients through social media: The benefits and challenges. Health Informatics Journal, 20(1), 50-58. DOI: $10.1177 / 1460458213476969$

Huang, E. y Dunbar, C.L. (2013). Connecting to patients via social media: A hype or a reality? Journal of Medical Marketing: Device, Diagnostic and Pharmaceutical Marketing, 13(1), 14-23. DOI: http://dx.doi.org/10.1177/1745790413477647 
Huesch, M., Currid-Halkett, E., y Doctor, J. (2014). Public hospital quality report awareness: evidence from National and Californian Internet searches and social media mentions, 2012. BMJ Open, 4(3). DOI: 10.1136/bmjopen-2013-004417

Jaakson, K. (2010). Management by values: are some values better than others? Journal of Management Development, 29(9), 795-806. DOI: $10.1108 / 02621711011072504$

Kemp, E., Jillapalli, R. y Becerra, E. (2014). Healthcare branding: developing emotionally based consumer brand relationships. Journal of Services Marketing, 28(2), 126-137. DOI: 10.1108/JSM-08-2012-0157

Lee, J., Choudhry, N., Wu, A., Matlin, O., Brennan, T. y Shrank, W. (2015). Patient Use of Email, Facebook, and Physician Websites to Communicate with Physicians: A National Online Survey of Retail Pharmacy Users. Journal of General Internal Medicine, 31(1), 45-51. DOI: 10.1007/s11606-015$3374-7$

McCarroll, M., Armbruster, S., Chung, J.E., Kim, J., McKenzie, A. y Von Gruenigen, V. (2014). Health Care and Social Media Platforms in Hospitals. Health Communication, 29(9), 947-952. DOI: 10.1080/10410236.2013.813831

Medina, P. (2011). Organización de la comunicación interna en hospitales. Madrid: Fragua. ISBN: 978-84-7074-445-7

Metzger, M., Flanagin, A. (2011). Using Web 2.0 Technologies to Enhance Evidence-Based Medical Information, Journal of Health Communication, 16 (sup1), 45-58. DOI: 10.1080/10810730.2011.589881

Ministerio de Sanidad, Política Social e Igualdad (2017). Catálogo Nacional de Hospitales. Madrid: Ministerio de Sanidad, Política Social e Igualdad.

Naveen, K., Anil, J. y Smruthi, T. (2014). Impact of healthcare marketing and branding on hospital services. International Journal of Research Foundation of Hospital \& Healthcare Administration, 2(1), 19-24. DOI: 10.5005/jp-journals-10035-1010

Nelson, W., Taylor, E. y Walsh, T. (2014). Building an Ethical Organizational Culture. The health care manager, 33(2), 158-164. DOI: 10.1097/HCM.0000000000000008

Park, H., Rodgers, S. y Stemmle, J. (2011). Health Organizations' Use of Facebook for Health Advertising and Promotion. Journal of Interactive Advertising, 12(1), 62-77. DOI: 10.1080/15252019.2011.10722191

Salathé, M., Freifeld, C., Mekaru, S., Tomasulo, A. y Brownstein, J. (2013). Influenza A (H7N9) and the importance of digital epidemiology. The New England Journal of Medicine, 369(5), 401-404. DOI: 10.1056/NEJMp1307752

Scott, D. (2010). The New Rules of Marketing and PR: How to Use Social Media, Blogs, News Releases, Online Video, and Viral Marketing to Reach Buyers Directly. Hoboken, NJ: John Wiley \& Sons. ISBN: 0470547812

Singal, A. y Jain, A. (2013). An empirical examination of the influence of corporate vision on internationalization. Strategic Change, 22(5-6), 243-257. DOI: $10.1002 / j s c .1937$.

Thaker S.I, Nowacki A.S, Mehta N.B y Edwards A.R. (2011). How U.S. hospitals use social media. Annals Internal Medicine, 154(10), 707-708. DOI: 10.7326/0003-4819-154-10-201105170-00021

The Change Foundation (2011). Using Social Media to Improve Healthcare Quality. Toronto: The Change Foundation.

Thielst, Ch. (2011). Using social media to engage patients: many tools exist to connect, communicate and build loyalty. Healthc Executive, 3, 66-70. Recuperado de http://thielst.typepad.com/files/satisfying-your-customers-with-social-media.pdf

Van de Belt, T., Berben, S., Samson, M., Engelen, L. y Schoonhoven, L. (2012). Use of social media by Western European hospitals: Iongitudinal study. Journal of medical Internet research, 14(3). DOI: 10.2196/jmir.1992

Van Riel, C., Fombrun, Ch. (2007). Essentials of corporate communication. New York : Routledge. ISBN: 1134335059

Visser, L., Bleijenbergh, I., Benschop, Y., Van Riel, A., y Bloem, B. (2016). Do online communities change power processes in healthcare? Using case studies to examine the use of online health communities by patients with Parkinson's disease. British Medical Journal, 6(11). DOI: 10.1136/bmjopen-2016-012110

Wright, K., Sparks, L. y O'Hair, D. (2008). Health communication in the 21st century. Malden: Blackwell Pub. ISBN: 9780470672723

Würz, A., Nurm, Ü.K. y Ekdahl, K. (2013). Enhancing the Role of Health Communication in the Prevention of Infectious Diseases. Journal of Health Communication, 18(12), 1566-1571. DOI: 10.1080/10810730.2013.840698

\section{Cómo citar este artículo en bibliografías - How to cite this article in bibliographies / references:}

GONZÁLEZ-PACANOWSKY, A.; MEDINA-AGUERREBERE P. (2018): "El rol de las redes sociales en la difusión de la identidad corporativa de los hospitales españoles". En Revista de la Asociación Española de Investigación de la Comunicación, vol. 5, número 10, pp. 30-38. 